\title{
Antibacterial mechanisms of silver nanoparticles on Pseudomonas aeruginosa
}

Xueting Yan, Bin $\mathrm{He}^{*}$, Ligang Hu, Guibin Jiang

State Key Laboratory of Environmental Chemistry and Ecotoxicology, Research Center for Eco-Environmental Sciences, Chinese Academy of Sciences, Beijing 100085, China

Silver nanoparticles (AgNPs) have been widely used due to their broad spectrum of antibacterial properties, effective sterilization and high safety. Studies had demonstrated that the antibiotic action of AgNPs is in a similar way to silver ions. However, the effectiv concentrations of AgNPs are three orders of mangitude lower than that of silver ions. The antibacterial mechanism of AgNPs has not been fully eluicidated from molecular level.

In our stury the mechanisms of antimicrobial activity of AgNPs in P. aeruginosa were investigated using metalloproteomics approach. The bacteria were cultured to stationary phase and exposed to $1.2 \mu \mathrm{g} \mathrm{mL}-1$ of AgNPs at $37{ }^{\circ} \mathrm{C}$ for $24 \mathrm{~h}$ in dark. After incubation, the bacteria were harvested and washed by PBS. Then the resuspended cell pellets were lysed and prepared for iTRAQ and 2D-LC-MS/MS analysis. The analytical results suggested that interference with the cell-membrane function and generation of intracellular reactive oxygen species (ROS) were the main pathways for the antibacterial activity of AgNPs, for which both the nanoparticles themselves and the silver ions released from AgNPs play a crucial role. The metalloproteomics and bioinformatic analysis of silver-binding and silver-regulated proteins identified many preteins pivotal in function of membrane stabilization, ATP synthesis, phospholipid syntheis and antibiotic resistance.

Acknowledgments We thank the financial support. from the National Natural Science Foundation of China (Projects 21677153, 21577153, 21777179 and 21522706) . 
This abstract is too long to be accepted for publication.

Please revise it so that it fits into the column on one page. 